\title{
Fixed Point Results of Contractive Mappings by Altering Distances and C-Class Functions in $b$-Dislocated Metric Spaces
}

\author{
Jani Dine' ${ }^{1}$, Kastriot Zoto' ${ }^{1}$, Arslan H. Ansari ${ }^{2}$ \\ ${ }^{1}$ Department of Mathematics and Computer Sciences, Faculty of Natural Sciences, University of Gjirokastra, Gjirokastra, Albania \\ ${ }^{2}$ Department of Mathematics, Karaj Branch, Islamic Azad University, Karaj, Iran \\ Email: jani_dine@yahoo.com, zotokastriot@yahoo.com, aminansari7@yahoo.com
}

How to cite this paper: Dine, J., Zoto, K. and Ansari, A.H. (2018) Fixed Point Results of Contractive Mappings by Altering Distances and C-Class Functions in $b$-Dislocated Metric Spaces. Open Access Library Journal, 5: e4657.

https://doi.org/10.4236/oalib.1104657

Received: May 13, 2018

Accepted: July 16, 2018

Published: July 19, 2018

Copyright $\odot 2018$ by authors and Open Access Library Inc.

This work is licensed under the Creative Commons Attribution International License (CC BY 4.0).

http://creativecommons.org/licenses/by/4.0/

\begin{abstract}
In this work, we recall definition of functions called as C-class and use the concepts of dislocated metric, b-dislocated metric, altering distance function. We prove some coincidence, fixed and common fixed point results for two pairs of weakly compatible mappings under $f-(\psi, \phi, s)$-contractive conditions and contractive conditions depended on another function $T$. Our theorems extend and generalize related results in the literature.
\end{abstract}

\section{Subject Areas}

Functional Analysis, Mathematical Analysis

\section{Keywords}

$f-(\psi, \phi, s)$ Contraction, C-Class Functions, $b_{d}$-Dislocated Metric Space, Coincidence Point, Common Fixed, Altering Distance Functions, Weakly Compatible Maps

\section{Introduction}

The study of metric fixed point theory in dislocated metric spaces was considered by P. Hitzler and A. K. Seda in [1] who introduced this metric as a generalization of usual metric, and generalized the Banach contraction principle on this space. Since then a lot of papers have been written on this topic treating the problem of existence and uniqueness of fixed points for mappings satisfying different contractive conditions, see [2]-[14]. N. Hussain et al. in [15] introduced the $b$-dislocated metric spaces associated with some topological aspects and properties. These spaces can be seen as generalizations of dislocated metric 
spaces and also as generalization of $b$-metric space introduced by Bakhtin in [16] and extensively used by Czerwik in [8]. Recently, there are many papers on existence and uniqueness of fixed point and common fixed point for one, two or more mappings under different types of contractive conditions in the setting of dislocated spaces and $b$-dislocated metric spaces.

Since altering distance functions were introduced by Khan et al. in [17], the study of the existence of fixed points of contractive maps in metric spaces and generalized metric spaces has a lot of interest for many authors which are based on this category of functions (see [17]-[23]). In September 2014, a class of functions called as C-class is presented by A. H. Ansari, see in [24] [25] and is important, see example 2.15 .

The present paper is organized in two sections. Using concepts mentioned above, in the first section, we develop some coincidence and common fixed point theorems (existence and uniqueness) for two pairs of weakly compatible mappings in the framework of $b_{d}$-dislocated metric space, using weak generalized $f-(\psi, \phi, s)$ contractive conditions. In the second section, we prove common fixed point theorems for a pair of mappings using generalized $f-(\psi, \phi, s)$ contractive condition and the concept of $T$-contractions. The related results generalize and improve various theorems in recent literature.

\section{Preliminaries}

Consistent with [1] and [15], the following definitions, notations, basic lemma and remarks will be needed in the sequel.

Definition 2.1 [1] Let $X$ be a nonempty set and a mapping $d_{l}: X \times X \rightarrow[0, \infty)$ is called a dislocated metric (or simply $d_{l}$-metric) if the following conditions hold for any $x, y, z \in X$ :

1) If $d_{l}(x, y)=0$, then $x=y$

2) $d_{l}(x, y)=d_{l}(y, x)$

3) $d_{l}(x, y) \leq d_{l}(x, z)+d_{l}(z, y)$

The pair $\left(X, d_{l}\right)$ is called a dislocated metric space (or $d$-metric space for short). Note that for $x=y, d_{l}(x, y)$ may not be 0 .

Definition 2.2 [15] Let $X$ be a nonempty set and a mapping $b_{d}: X \times X \rightarrow[0, \infty)$ is called a $b$-dislocated metric (or simply $b_{d}$-dislocated metric) if the following conditions hold for any $x, y, z \in X$ and $s \geq 1$ :

1) If $b_{d}(x, y)=0$, then $x=y$

2) $b_{d}(x, y)=b_{d}(y, x)$

3) $b_{d}(x, y) \leq s\left[b_{d}(x, z)+b_{d}(z, y)\right]$

The pair $\left(X, b_{d}\right)$ is called a $b$-dislocated metric space. And the class of $b$-dislocated metric space is larger than that of dislocated metric spaces, since a $b$-dislocated metric is a dislocated metric when $s=1$.

Example 2.3 If $X=R$, then $d_{l}(x, y)=|x|+|y|$ defines a dislocated metric on $X$.

Definition 2.4 [1] A sequence $\left(x_{n}\right)$ in $d_{l}$-metric space $\left(X, d_{l}\right)$ is called: 
1) a Cauchy sequence if, for given $\varepsilon>0$, there exists $n_{0} \in N$ such that for all $m, n \geq n_{0}$, we have or $\lim _{n, m \rightarrow \infty} d_{l}\left(x_{n}, x_{m}\right)=0$;

2) convergent with respect to $d_{l}$ if there exists $x \in X$ such that $d_{l}\left(x_{n}, x\right) \rightarrow 0$ as $n \rightarrow \infty$. In this case, $x$ is called the limit of $\left(x_{n}\right)$ and we write $x_{n} \rightarrow x$.

A $d_{l}$-metric space $X$ is called complete if every Cauchy sequence in $X$ converges to a point in $X$.

In [15], it was shown that each $b_{d}$-metric on $X$ generates a topology $\tau_{b_{d}}$ whose base is the family of open $b_{d}$-balls $B_{b_{d}}(x, \varepsilon)=\left\{y \in X: b_{d}(x, y)<\varepsilon\right\}$.

Also in [15], there are presented some topological properties of $b_{d}$-metric spaces.

Definition 2.5 [15] Let $\left(X, b_{d}\right)$ be a $b_{d}$-metric space, and $\left\{x_{n}\right\}$ be a sequence of points in $X$. A point $x \in X$ is said to be the limit of the sequence $\left\{x_{n}\right\}$ if $\lim _{n \rightarrow \infty} b_{d}\left(x_{n}, x\right)=0$ and we say that the sequence $\left\{x_{n}\right\}$ is $b_{d}$ -convergent to $x$ and denote it by $x_{n} \rightarrow x$ as $n \rightarrow \infty$.

The limit of a $b_{d}$-convergent sequence in a $b_{d}$-metric space is unique ([15], Proposition 1.27).

Definition 2.6 [15] A sequence $\left\{x_{n}\right\}$ in a $b_{d}$-metric space $\left(X, b_{d}\right)$ is called a $b_{d}$-Cauchy sequence if, given $\varepsilon>0$, there exists $n_{0} \in N$ such that for all $n, m>n_{0}$, we have $b_{d}\left(x_{n}, x_{m}\right)<\varepsilon$ or $\lim _{n, m \rightarrow \infty} b_{d}\left(x_{n}, x_{m}\right)=0$. Every $b_{d}$ -convergent sequence in a $b_{d}$-metric space is a $b_{d}$-Cauchy sequence.

Remark 2.7 The sequence $\left\{x_{n}\right\}$ in a $b_{d}$-metric space $\left(X, b_{d}\right)$ is called a $b_{d}$-Cauchy sequence if $\lim _{n, m \rightarrow \infty} b_{d}\left(x_{n}, x_{n+p}\right)=0$ for all $p \in N^{*}$.

Definition 2.8 [15] $\mathrm{A} b_{d}^{n, m \rightarrow \infty}$-metric space $\left(X, b_{d}\right)$ is called complete if every $b_{d}$-Cauchy sequence in $X$ is $b_{d}$-convergent.

Example 2.9 If $X=R^{+} \cup\{0\}$, then $b_{d}(x, y)=(x+y)^{2}$ defines a $b$-dislocated metric on $X$ with parameter $s=2$.

Example 2.10 Let $X=R^{+} \cup\{0\}$ and any constant $\alpha>0$. Define function $d_{l}: X \times X \rightarrow R^{+}$by $d_{l}(x, y)=\alpha(x+y)$. Then, the pair $\left(X, d_{l}\right)$ is a dislocated metric space.

If $F x=S x$ for some $x \in X$, then $x$ is called the coincidence point of $F$ and $S$. Furthermore, if the mappings commute at each coincidence point, then such mappings are called weakly compatible [4].

Definition 2.11 [17] The altering distances functions $\psi$ and $\varphi$ are defined as

$$
\begin{gathered}
\Psi=\{\psi:[0, \infty) \rightarrow[0, \infty) / \psi \text { is continuous, nondecreasing, and } \psi(t)=0 \text { if } t=0\} \\
\Phi=\{\phi:[0, \infty) \rightarrow[0, \infty) / \phi \text { is lower semicontinuous, and } \phi(t)=0 \text { if } t=0\}
\end{gathered}
$$

The following lemmas are used to prove our results.

Lemma 2.12 Let $\left(X, b_{d}\right)$ be a $b$-dislocated metric space with parameter $s \geq 1$. Then

1) If $b_{d}(x, y)=0$ then $b_{d}(x, x)=b_{d}(y, y)=0$;

2) If $\left(x_{n}\right)$ is a sequence such that $\lim _{n \rightarrow \infty} b_{d}\left(x_{n}, x_{n+1}\right)=0$, then we have 


$$
\lim _{n \rightarrow \infty} b_{d}\left(x_{n}, x_{n}\right)=\lim _{n \rightarrow \infty} b_{d}\left(x_{n+1}, x_{n+1}\right)=0 ;
$$

3) If $x \neq y$, then $b_{d}(x, y)>0$;

Proof. It is clear.

Lemma 2.13 [15] Let $\left(X, b_{d}\right)$ be a $b$-dislocated metric space with parameter $s \geq 1$. Suppose that $\left\{x_{n}\right\}$ and $\left\{y_{n}\right\}$ are $b_{d}$-convergent to $x, y \in X$, respectively. Then we have

$$
\frac{1}{s^{2}} b_{d}(x, y) \leq \liminf _{n \rightarrow \infty} b_{d}\left(x_{n}, y_{n}\right) \leq \lim _{n \rightarrow \infty} \sup b_{d}\left(x_{n}, y_{n}\right) \leq s^{2} b_{d}(x, y)
$$

In particular, if $b_{d}(x, y)=0$, then we have $\lim _{n \rightarrow \infty} b_{d}\left(x_{n}, y_{n}\right)=0=b_{d}(x, y)$. Moreover, for each $z \in X$, we have

$$
\frac{1}{S} b_{d}(x, z) \leq \liminf _{n \rightarrow \infty} b_{d}\left(x_{n}, z\right) \leq \lim _{n \rightarrow \infty} \sup b_{d}\left(x_{n}, z\right) \leq s b_{d}(x, z)
$$

In particular, if $b_{d}(x, z)=0$, then we have $\lim _{n \rightarrow \infty} b_{d}\left(x_{n}, z\right)=0=b_{d}(x, z)$.

Definition 2.14. [24] [25] We say that a function $f:[0, \infty)^{2} \rightarrow R$ is called a $C$-class function if it is continuous and satisfies the following properties.

$$
\begin{aligned}
& \text { 1) } f(s, t) \leq s \\
& \text { 2) } f(s, t)=s \Rightarrow s=0 \text { or } t=0 \text { for all } s, t \in[0, \infty) \\
& \text { 3) } f(0,0)=0
\end{aligned}
$$

We denote $C$-class functions as $C$.

Example 2.15 [24] [25] The following functions $f:[0, \infty)^{2} \rightarrow R$ are elements of $C$, for all $s, t \in[0, \infty)$ :

1) $f(s, t)=s-t, f(s, t)=s \Rightarrow t=0$

2) $f(s, t)=\frac{s-t}{1+t}, f(s, t)=s \Rightarrow t=0$

3) $f(s, t)=\frac{s t}{1+t}, f(s, t)=s \Rightarrow s=0$

4) $f(s, t)=\frac{s}{1+t}, f(s, t)=s \Rightarrow s=0$ or $t=0$

5) $f(s, t)=\log \frac{t+a^{s}}{1+t}, a>1, f(s, t)=s \Rightarrow s=0$ or $t=0$

For $t=1$, we have $f(s, 1)=\ln \frac{1+a^{s}}{2}, a>e, f(s, 1)=s \Rightarrow s=0$

6) $f(s, t)=(s+k)^{\frac{1}{1+t}}-k, k>1, f(s, t)=s \Rightarrow t=0$

7) $f(s, t)=s \log _{a+t} a, a>1, f(s, t)=s \Rightarrow s=0$ or $t=0$

8) $f(s, t)=m s, 0<m<1 ; f(s, t)=s \Rightarrow s=0$

9) $f(s, t)=\phi(s), f(s, t)=s \Rightarrow s=0$, here $\phi:[0, \infty) \rightarrow[0, \infty)$ is continuous and such that $\phi(0)=0$ and $\phi(t)<t$ for $t>0$. 


\section{Main Results}

Before we give the main result we denote with letter $N(x, y)$ the following set

$$
N(x, y)=\max \left\{b_{d}(S x, T y), b_{d}(F x, S x), b_{d}(G y, T y), b_{d}(S x, G y), b_{d}(F x, T y)\right\}(3.1 .1)
$$

for all $x, y \in X$.

Motivated by the works of [15] [21]-[29] we extend the concept of $(\psi, \phi)$ -weakly contractive maps to four maps in a $b$-dislocated metric space, giving the following definition.

Definition 3.1 Let $F, G, S, T$ be four self maps of a $b$-dislocated metric space $\left(X, b_{d}\right)$ with parameter $s \geq 1$. If there exists $\psi \in \Psi, \varphi \in \Phi$ and $f \in C$ such that

$$
\psi\left(2 s^{4} b_{d}(F x, G y)\right) \leq f(\psi(N(x, y)), \phi(N(x, y)))
$$

for all $x, y \in X$, where $N(x, y)$ is defined as in (3.1.1) then $F, G, S$ and $T$ are said to satisfy a generalized $f-(\psi, \phi, s)$ weakly contractive condition.

Theorem 3.2 Let $\left(X, b_{d}\right)$ be a $b$-dislocated metric space with parameter $s \geq 1$ and $S, T, F, G: X \rightarrow X$ are self-mappings such that (a) $F(X) \subset T(X)$, $G(X) \subset S(X)$ and satisfy generalized $f-(\psi, \phi, s)$ weakly contractive condition. If one of $F(X), S(X), T(X)$ or $G(X)$ is a complete subspace of $X$, then $(F, S)$ and $(G, T)$ have a point of coincidence in $X$. Moreover if suppose that $(F, S)$ and $(G, T)$ are weakly compatible pairs, then $F, G, S, T$ have a unique common fixed point.

Proof. Let $x_{0}$ be an arbitrary point in $X$. Since $F(X) \subset T(X)$ we can choose $x_{1} \in X$ such that $y_{0}=f x_{0}=T x_{1}$. And since $G(X) \subset S(X)$ corresponding to $x_{1} \in X$ we can choose $x_{2} \in X$ such that $y_{1}=G x_{1}=S x_{2}$. Continuing the same process we obtain sequences $\left\{x_{n}\right\}$ and $\left\{y_{n}\right\}$ in $X$ such that:

$$
y_{2 n}=F x_{2 n}=T x_{2 n+1} ; y_{2 n+1}=G x_{2 n+1}=S x_{2 n+2} \text { for all } n=0,1,2, \cdots
$$

We consider following steps:

Step 1. If $y_{2 n}=y_{2 n+1}$ (that means $\left.b_{d}\left(y_{2 n}, y_{2 n+1}\right)=0\right)$ for some $n$, then $G x_{2 n+1}=T x_{2 n+1}$. Hence $x_{2 n+1}$ is a coincidence point of $G$ and $T$. Using definition of $N(x, y)$ and lemma 2.12 we have,

$$
\begin{aligned}
N\left(x_{2 n+2}, x_{2 n+1}\right) & \\
= & \max \left\{b_{d}\left(S x_{2 n+2}, T x_{2 n+1}\right), b_{d}\left(F x_{2 n+2}, S x_{2 n+2}\right), b_{d}\left(G x_{2 n+1}, T x_{2 n+1}\right),\right. \\
& \left.b_{d}\left(S x_{2 n+2}, G x_{2 n+1}\right), b_{d}\left(F x_{2 n+2}, T x_{2 n+1}\right)\right\} \\
= & \max \left\{b_{d}\left(y_{2 n+1}, y_{2 n}\right), b_{d}\left(y_{2 n+2}, y_{2 n+1}\right), b_{d}\left(y_{2 n+1}, y_{2 n}\right),\right. \\
& \left.\quad b_{d}\left(y_{2 n+1}, y_{2 n+1}\right), b_{d}\left(y_{2 n+2}, y_{2 n}\right)\right\} \\
\leq & \max \left\{b_{d}\left(y_{2 n+1}, y_{2 n}\right), b_{d}\left(y_{2 n+2}, y_{2 n+1}\right), b_{d}\left(y_{2 n+1}, y_{2 n}\right),\right. \\
& \left.\quad 2 s b_{d}\left(y_{2 n+1}, y_{2 n}\right), s\left[b_{d}\left(y_{2 n+2}, y_{2 n+1}\right)+b_{d}\left(y_{2 n+1}, y_{2 n}\right)\right]\right\} \\
= & \max \left\{0, b_{d}\left(y_{2 n+2}, y_{2 n+1}\right), 0,0, s b_{d}\left(y_{2 n+2}, y_{2 n+1}\right)\right\} \\
= & s b_{d}\left(y_{2 n+2}, y_{2 n+1}\right)
\end{aligned}
$$


Thus $N\left(x_{2 n+2}, x_{2 n+1}\right) \leq s b_{d}\left(y_{2 n+2}, y_{2 n+1}\right)$

Using condition (A) and property of C-class, we have that

$$
\begin{aligned}
\psi\left(s b_{d}\left(y_{2 n+1}, y_{2 n+2}\right)\right) & =\psi\left(s b_{d}\left(G x_{2 n+1}, F x_{2 n+2}\right)\right) \\
& =\psi\left(2 s b_{d}\left(F x_{2 n+2}, G x_{2 n+1}\right)\right) \\
& \leq \psi\left(2 s^{4} b_{d}\left(F x_{2 n+2}, G x_{2 n+1}\right)\right) \\
& \leq f\left(\psi\left(N\left(x_{2 n+2}, x_{2 n+1}\right)\right), \phi\left(N\left(x_{2 n+2}, x_{2 n+1}\right)\right)\right) \\
& \leq \psi\left(N\left(x_{2 n+2}, x_{2 n+1}\right)\right)
\end{aligned}
$$

By property of $\psi$ we have,

$$
s b_{d}\left(y_{2 n+2}, y_{2 n+1}\right) \leq N\left(x_{2 n+2}, x_{2 n+1}\right)
$$

As a result we get,

$$
N\left(x_{2 n+2}, x_{2 n+1}\right)=s b_{d}\left(y_{2 n+1}, y_{2 n+2}\right) .
$$

Again from contractive condition of theorem have,

$$
\begin{aligned}
\psi\left(s b_{d}\left(y_{2 n+2}, y_{2 n+1}\right)\right) & =\psi\left(s b_{d}\left(F x_{2 n+2}, G x_{2 n+1}\right)\right) \\
& \leq \psi\left(2 s^{4} b_{d}\left(F x_{2 n+2}, G x_{2 n+1}\right)\right) \\
& \leq f\left(\psi\left(N\left(x_{2 n+2}, x_{2 n+1}\right)\right), \phi\left(N\left(x_{2 n+2}, x_{2 n+1}\right)\right)\right) \\
& =f\left(\psi\left(s b_{d}\left(y_{2 n+1}, y_{2 n+2}\right)\right), \phi\left(s b_{d}\left(y_{2 n+1}, y_{2 n+2}\right)\right)\right) \\
& \leq \psi\left(s b_{d}\left(y_{2 n+1}, y_{2 n+2}\right)\right)
\end{aligned}
$$

The inequality above implies,

$$
f\left(\psi\left(s b_{d}\left(y_{2 n+1}, y_{2 n+2}\right)\right), \phi\left(s b_{d}\left(y_{2 n+1}, y_{2 n+2}\right)\right)\right)=\psi\left(s b_{d}\left(y_{2 n+1}, y_{2 n+2}\right)\right)
$$

By property of function $f$ of C-class we obtain

$$
\psi\left(s b_{d}\left(y_{2 n+1}, y_{2 n+2}\right)\right)=0 \text { or } \phi\left(s b_{d}\left(y_{2 n+1}, y_{2 n+2}\right)\right)=0 \text {. }
$$

And also by property of $\psi, \phi$ we get $b_{d}\left(y_{2 n+1}, y_{2 n+2}\right)=0$ so that $y_{2 n+1}=y_{2 n+2}$ and then $F x_{2 n+2}=S x_{2 n+2}$. Also $x_{2 n+2}$ is a coincidence point of $F$ and $S$.

Step 2. Suppose $y_{2 n} \neq y_{2 n+1}$ that means $b_{d}\left(y_{2 n}, y_{2 n+1}\right)>0$ for all $n$ by condition (3.1.1) we have:

$$
\begin{aligned}
& \begin{aligned}
N\left(x_{2 n}, x_{2 n+1}\right)= & \max \left\{b_{d}\left(S x_{2 n}, T x_{2 n+1}\right), b_{d}\left(F x_{2 n}, S x_{2 n}\right), b_{d}\left(G x_{2 n+1}, T x_{2 n+1}\right)\right. \\
& \left.b_{d}\left(S x_{2 n}, G x_{2 n+1}\right), b_{d}\left(F x_{2 n}, T x_{2 n+1}\right)\right\}
\end{aligned} \\
& =\max \left\{b_{d}\left(y_{2 n-1}, y_{2 n}\right), b_{d}\left(y_{2 n}, y_{2 n-1}\right), b_{d}\left(y_{2 n+1}, y_{2 n}\right)\right. \\
& \left.b_{d}\left(y_{2 n-1}, y_{2 n+1}\right), b_{d}\left(y_{2 n}, y_{2 n}\right)\right\}
\end{aligned}
$$


Also from condition of theorem we have:

$$
\begin{aligned}
\psi\left(2 s b_{d}\left(y_{2 n}, y_{2 n+1}\right)\right) & \leq \psi\left(2 s^{4} b_{d}\left(y_{2 n}, y_{2 n+1}\right)\right) \\
& =\psi\left(2 s^{4} b_{d}\left(F x_{2 n}, G x_{2 n+1}\right)\right) \\
& \leq f\left(\psi\left(N\left(x_{2 n}, x_{2 n+1}\right)\right), \phi\left(N\left(x_{2 n}, x_{2 n+1}\right)\right)\right) \\
& \leq \psi\left(N\left(x_{2 n}, x_{2 n+1}\right)\right)
\end{aligned}
$$

By property of function $\psi$ we have

$$
2 s b_{d}\left(y_{2 n}, y_{2 n+1}\right) \leq N\left(x_{2 n}, x_{2 n+1}\right)
$$

From (3.2.3) and (3.2.4) we get

$$
N\left(x_{2 n}, x_{2 n+1}\right)=2 s b_{d}\left(y_{2 n}, y_{2 n+1}\right)
$$

Also from condition of theorem and (3.2.5) we have,

$$
\begin{aligned}
\psi\left(2 s b_{d}\left(y_{2 n}, y_{2 n+1}\right)\right) & \leq \psi\left(2 s^{4} b_{d}\left(y_{2 n}, y_{2 n+1}\right)\right) \\
& =\psi\left(2 s^{4} b_{d}\left(F x_{2 n}, G x_{2 n+1}\right)\right) \\
& \leq f\left(\psi\left(N\left(x_{2 n}, x_{2 n+1}\right)\right), \phi\left(N\left(x_{2 n}, x_{2 n+1}\right)\right)\right) \\
& =f\left(\psi\left(2 s b_{d}\left(y_{2 n}, y_{2 n+1}\right)\right), \phi\left(2 s b_{d}\left(y_{2 n}, y_{2 n+1}\right)\right)\right) \\
& \leq \psi\left(2 s b_{d}\left(y_{2 n}, y_{2 n+1}\right)\right)
\end{aligned}
$$

The above inequality implies:

$$
\begin{aligned}
\psi\left(2 s b_{d}\left(y_{2 n}, y_{2 n+1}\right)\right) & \leq f\left(\psi\left(2 s b_{d}\left(y_{2 n}, y_{2 n+1}\right)\right), \phi\left(2 s b_{d}\left(y_{2 n}, y_{2 n+1}\right)\right)\right) \\
& \leq \psi\left(2 s b_{d}\left(y_{2 n}, y_{2 n+1}\right)\right)
\end{aligned}
$$

which means

$$
f\left(\psi\left(2 s b_{d}\left(y_{2 n}, y_{2 n+1}\right)\right), \phi\left(2 s b_{d}\left(y_{2 n}, y_{2 n+1}\right)\right)\right)=\psi\left(2 s b_{d}\left(y_{2 n}, y_{2 n+1}\right)\right) \text {. }
$$

From property of C-class we obtain

$$
\psi\left(2 s b_{d}\left(y_{2 n}, y_{2 n+1}\right)\right)=0 \text { or } \phi\left(2 s b_{d}\left(y_{2 n}, y_{2 n+1}\right)\right)=0 \text {. }
$$

So we have $b_{d}\left(y_{2 n}, y_{2 n+1}\right)=0$ that is a contradiction since we suppose $b_{d}\left(y_{2 n}, y_{2 n+1}\right)>0$.

So we have $b_{d}\left(y_{2 n}, y_{2 n+1}\right) \leq b_{d}\left(y_{2 n-1}, y_{2 n}\right)$.

In a similar way as above we have $b_{d}\left(y_{2 n+1}, y_{2 n+2}\right) \leq b_{d}\left(y_{2 n}, y_{2 n+1}\right)$. As a result the sequence $\left\{b_{d}\left(y_{n}, y_{n+1}\right)\right\}$ is non increasing and bounded below. And so there exists $l \geq 0$ such that,

$$
\lim _{n \rightarrow \infty} b_{d}\left(y_{n}, y_{n+1}\right)=\lim _{n \rightarrow \infty} N\left(x_{n}, x_{n+1}\right)=l .
$$

Suppose that $l>0$. Since $\psi$ is continuous and $\phi$ is lower semi continuous we have:

$$
\psi(l)=\lim _{n \rightarrow \infty} \psi\left(N\left(x_{n}, x_{n+1}\right)\right) \text { and } \phi(l) \leq \liminf _{n \rightarrow \infty} \phi\left(N\left(x_{n}, x_{n+1}\right)\right)
$$

If we consider condition (A) we have, 


$$
\begin{aligned}
\psi\left(2 s b_{d}\left(y_{2 n+2}, y_{2 n+1}\right)\right) & \leq \psi\left(2 s^{4} b_{d}\left(y_{2 n+2}, y_{2 n+1}\right)\right) \\
& \leq f\left(\psi\left(N\left(x_{2 n+2}, x_{2 n+1}\right)\right), \phi\left(N\left(x_{2 n+2}, x_{2 n+1}\right)\right)\right) \\
& \leq \psi\left(N\left(x_{2 n+2}, x_{2 n+1}\right)\right)
\end{aligned}
$$

taking the upper limit as $n \rightarrow \infty$ in (3.2.7) and using (3.2.6) we have that,

$$
\begin{aligned}
& \psi(2 s l) \leq f(\psi(2 s l), \phi(2 s l)) \leq \psi(2 s l) \\
& \Rightarrow f(\psi(2 s l), \phi(2 s l))=\psi(2 s l) \\
& \Rightarrow \psi(2 s l)=0 \text { or } \phi(2 s l)=0 .
\end{aligned}
$$

From (3.2.8) and property of $\psi$ we get $l=0$ that is a contradiction. Hence

$$
\lim _{n \rightarrow \infty} b_{d}\left(y_{n}, y_{n+1}\right)=\lim _{n \rightarrow \infty} N\left(x_{n}, x_{n+1}\right)=0
$$

Now we prove that $\left\{y_{n}\right\}$ is a $b_{d}$-Cauchy sequence. Assume the contrary that $\left\{y_{2 n}\right\}$ is not a $b_{d}$-Cauchy sequence. Then there exists $\varepsilon>0$ for which we can find subsequences $\left\{y_{2 m_{k}}\right\}$ and $\left\{y_{2 n_{k}}\right\}$ of $\left\{y_{2 n}\right\}$ so that $n_{k}$ is the smallest index for which $2 n_{k}>2 m_{k}>k$, that

$$
\begin{array}{r}
b_{d}\left(y_{2 m_{k}}, y_{2 n_{k}}\right) \geq \varepsilon \\
\text { and } b_{d}\left(y_{2 m_{k}}, y_{2 n_{k}-2}\right)<\varepsilon
\end{array}
$$

From property c) of definition 2.2 we have:

$$
\begin{aligned}
\varepsilon & \leq b_{d}\left(y_{2 m_{k}}, y_{2 n_{k}}\right) \leq s b_{d}\left(y_{2 m_{k}}, y_{2 n_{k}-2}\right)+s b_{d}\left(y_{2 n_{k}-2}, y_{2 n_{k}}\right) \\
& \leq s b_{d}\left(y_{2 m_{k}}, y_{2 n_{k}-2}\right)+s^{2} b_{d}\left(y_{2 n_{k}-2}, y_{2 n_{k}-1}\right)+s^{2} b_{d}\left(y_{2 n_{k}-1}, y_{2 n_{k}}\right)
\end{aligned}
$$

Taking the upper limit as $k \rightarrow \infty$ in (3.2.12) and using result (3.129) and (3.2.11), we get

$$
\varepsilon \leq \lim _{k \rightarrow \infty} \sup b_{d}\left(y_{2 m_{k}}, y_{2 n_{k}}\right) \leq \varepsilon S
$$

Also we have

$$
\varepsilon \leq b_{d}\left(y_{2 m_{k}}, y_{2 n_{k}}\right) \leq s b_{d}\left(y_{2 m_{k}}, y_{2 n_{k}-1}\right)+s b_{d}\left(y_{2 n_{k}-1}, y_{2 n_{k}}\right)
$$

Hence taking the upper limit in above inequality, we obtain

$$
\frac{\varepsilon}{S} \leq \limsup _{k \rightarrow \infty} b_{d}\left(y_{2 m_{k}}, y_{2 n_{k}-1}\right)
$$

Again from property $c$ ) of definition 2.2, we have

$$
b_{d}\left(y_{2 m_{k}}, y_{2 n_{k}-1}\right) \leq s b_{d}\left(y_{2 m_{k}}, y_{2 n_{k}}\right)+s b_{d}\left(y_{2 n_{k}}, y_{2 n_{k}-1}\right)
$$

Thus from 3.2.9; 3.2.15 we have

$$
\lim _{k \rightarrow \infty} \sup b_{d}\left(y_{2 m_{k}}, y_{2 n_{k}-1}\right) \leq \varepsilon s^{2}
$$

As a result,

$$
\frac{\varepsilon}{S} \leq \lim _{k \rightarrow \infty} \sup b_{d}\left(y_{2 m_{k}}, y_{2 n_{k}-1}\right) \leq \varepsilon s^{2}
$$


Similarly,

$$
\varepsilon \leq b_{d}\left(y_{2 m_{k}}, y_{2 n_{k}}\right) \leq s b_{d}\left(y_{2 m_{k}}, y_{2 m_{k}+1}\right)+s b_{d}\left(y_{2 m_{k}+1}, y_{2 n_{k}}\right)
$$

Taking the upper limit in (3.2.19) and using 3.2.9, we get

$$
\frac{\varepsilon}{s} \leq \limsup _{k \rightarrow \infty} b_{d}\left(y_{2 m_{k}+1}, y_{2 n_{k}}\right)
$$

Similarly,

$$
\begin{aligned}
\varepsilon & \leq b_{d}\left(y_{2 m_{k}}, y_{2 n_{k}}\right) \leq s b_{d}\left(y_{2 m_{k}}, y_{2 m_{k}+1}\right)+s b_{d}\left(y_{2 m_{k}+1}, y_{2 n_{k}}\right) \\
& \leq s b_{d}\left(y_{2 m_{k}}, y_{2 m_{k}+1}\right)+s^{2} b_{d}\left(y_{2 m_{k}+1}, y_{2 n_{k}-1}\right)+s^{2} b_{d}\left(y_{2 n_{k}-1}, y_{2 n_{k}}\right)
\end{aligned}
$$

Taking the upper limit in above inequality and using (3.2.9), we have

$$
\frac{\varepsilon}{s^{2}} \leq \limsup _{k \rightarrow \infty} b_{d}\left(y_{2 m_{k}+1}, y_{2 n_{k}-1}\right)
$$

Also,

$$
b_{d}\left(y_{2 m_{k}+1}, y_{2 n_{k}-1}\right) \leq s b_{d}\left(y_{2 m_{k}+1}, y_{2 m_{k}}\right)+s b_{d}\left(y_{2 m_{k}}, y_{2 n_{k}-1}\right)
$$

Taking the upper limit and using 3.2.9; 3.2 .18 we get

$$
\limsup _{k \rightarrow \infty} b_{d}\left(y_{2 m_{k}+1}, y_{2 n_{k}-1}\right) \leq \varepsilon s^{3}
$$

So, by (3.2.21) and (3.2.22) we have

$$
\frac{\varepsilon}{s^{2}} \leq \limsup _{k \rightarrow \infty} b_{d}\left(y_{2 m_{k}+1}, y_{2 n_{k}-1}\right) \leq \varepsilon s^{3}
$$

According to the set (3.1.1) we have:

$$
\begin{aligned}
N\left(x_{2 n_{k}}, x_{2 m_{k}+1}\right)= & \max \left\{b_{d}\left(S x_{2 n_{k}}, T x_{2 m_{k}+1}\right), b_{d}\left(F x_{2 n_{k}}, S x_{2 n_{k}}\right), b_{d}\left(G x_{2 m_{k}+1}, T x_{2 m_{k}+1}\right),\right. \\
& \left.b_{d}\left(S x_{2 n_{k}}, G x_{2 m_{k}+1}\right), b_{d}\left(F x_{2 n_{k}}, T x_{2 m_{k}+1}\right)\right\} \\
=\max \{ & b_{d}\left(y_{2 n_{k}-1}, y_{2 m_{k}}\right), b_{d}\left(y_{2 n_{k}}, y_{2 n_{k}-1}\right), b_{d}\left(y_{2 m_{k}+1}, y_{2 m_{k}}\right), \\
& \left.b_{d}\left(y_{2 n_{k}-1}, y_{2 m_{k}+1}\right), b_{d}\left(y_{2 n_{k}}, y_{2 m_{k}}\right)\right\}
\end{aligned}
$$

Taking the upper limit in (3.2.24) and using results $3.2 .9 ; 3.2 .18 ; 3.2 .13 ; 3.2 .23$ we get

$$
\begin{aligned}
& \limsup _{k \rightarrow \infty} N\left(x_{2 n_{k}}, x_{2 m_{k}+1}\right) \\
& =\underset{k \rightarrow \infty}{\limsup \max }\left\{b_{d}\left(y_{2 n_{k}-1}, y_{2 m_{k}}\right), b_{d}\left(y_{2 n_{k}}, y_{2 n_{k}-1}\right), b_{d}\left(y_{2 m_{k}+1}, y_{2 m_{k}}\right),\right. \\
& \left.\qquad b_{d}\left(y_{2 n_{k}-1}, y_{2 m_{k}+1}\right), b_{d}\left(y_{2 n_{k}}, y_{2 m_{k}}\right)\right\} \\
& \leq \max \left\{\varepsilon s^{2}, 0,0, \varepsilon s, \varepsilon s^{3}\right\}
\end{aligned}
$$

Similarly, we can show,

$$
\min \left\{\frac{\varepsilon}{s}, \varepsilon, \frac{\varepsilon}{s^{2}}\right\} \leq \liminf _{k \rightarrow \infty} N\left(x_{2 n_{k}}, x_{2 m_{k}+1}\right) \leq \varepsilon s^{3}
$$

From contractive condition of theorem, we have 


$$
\begin{aligned}
\psi\left(2 s^{4} b_{d}\left(y_{2 n_{k}}, y_{2 m_{k}+1}\right)\right) & \leq \psi\left(2 s^{4} b_{d}\left(F x_{2 n_{k}}, G x_{2 m_{k}+1}\right)\right) \\
& \leq f\left(\psi\left(N\left(x_{2 n_{k}}, x_{2 m_{k}+1}\right)\right), \phi\left(N\left(x_{2 n_{k}}, x_{2 m_{k}+1}\right)\right)\right) \\
& \leq \psi\left(N\left(x_{2 n_{k}}, x_{2 m_{k}+1}\right)\right)
\end{aligned}
$$

Taking the upper limit as $k \rightarrow \infty$ in (3.2.27) and using 3.2.25; 3.2.26, we obtain

$$
\begin{aligned}
\psi\left(2 \varepsilon s^{3}\right) & =\psi\left(2 s^{4} \frac{\varepsilon}{s}\right) \leq \psi\left(2 s^{4} \limsup _{k \rightarrow \infty} b_{d}\left(y_{2 n_{k}}, y_{2 m_{k}+1}\right)\right) \\
& =\psi\left(2 s^{4} \limsup _{k \rightarrow \infty} b_{d}\left(F x_{2 n_{k}}, G x_{2 m_{k}+1}\right)\right) \\
& \leq f\left(\psi\left(\limsup _{k \rightarrow \infty} N\left(x_{2 n_{k}}, x_{2 m_{k}+1}\right)\right), \phi\left(\liminf _{k \rightarrow \infty} N\left(x_{2 n_{k}}, x_{2 m_{k}+1}\right)\right)\right) \\
& \leq f\left(\psi\left(\varepsilon s^{3}\right), \phi\left(\varepsilon s^{3}\right)\right) \leq \psi\left(\varepsilon s^{3}\right)
\end{aligned}
$$

From this inequality and since $\psi$ is non decreasing follows that

$$
2 \varepsilon s^{3} \leq \varepsilon s^{3} \Leftrightarrow \varepsilon s^{3}=0 \Leftrightarrow \varepsilon=0 .
$$

That is a contradiction since we supposed $\varepsilon>0$. Thus $\left\{y_{n}\right\}$ is a $b_{d}$-Cauchy sequence in b-dislocated metric space $\left(X, b_{d}\right)$. Also the subsequences $\left\{f x_{2 n}\right\}$, $\left\{T x_{2 n+1}\right\},\left\{G x_{2 n+1}\right\},\left\{S x_{2 n+2}\right\}$ are $b_{d}$-Cauchy. Let we suppose that $F(X)$ is a complete subspace of $X$, since the subsequence $\left\{f x_{2 n}\right\}$ is $b_{d}$-Cauchy then there exists $z \in F(X)$ such that $\lim _{n \rightarrow \infty} y_{2 n}=\lim _{n \rightarrow \infty} F x_{2 n}=z$. Then we have,

$$
\lim _{n \rightarrow \infty} T x_{2 n+1}=\lim _{n \rightarrow \infty} F x_{2 n}=\lim _{n \rightarrow \infty} G x_{2 n+1}=\lim _{n \rightarrow \infty} S x_{2 n+2}=z .
$$

Since $z \in F(X) \subset T(X)$, then there exists $y \in X$ such that $T y=z$. According to (3.1.1) consider

$$
\begin{aligned}
& N\left(x_{2 n}, y\right) \\
& =\max \left\{b_{d}\left(S x_{2 n}, T y\right), b_{d}\left(F x_{2 n}, S x_{2 n}\right), b_{d}(G y, T y), b_{d}\left(S x_{2 n}, G y\right), b_{d}\left(F x_{2 n}, T y\right)\right\} \\
& =\max \left\{b_{d}\left(y_{2 n-1}, z\right), b_{d}\left(y_{2 n}, y_{2 n-1}\right), b_{d}(G y, z), b_{d}\left(y_{2 n-1}, G y\right), b_{d}\left(y_{2 n}, z\right)\right\}
\end{aligned}
$$

Taking the upper limit and using lemma 2.13, result (3.2.9) and (3.2.28) we obtain

$$
\lim _{n \rightarrow \infty} \sup N\left(x_{2 n}, y\right) \leq s b_{d}(G y, z)
$$

Using contractive condition (A) of theorem we have,

$$
\begin{aligned}
\psi\left(2 s^{4} b_{d}\left(F x_{2 n}, G y\right)\right) & \leq f\left(\psi\left(N\left(x_{2 n}, y\right)\right), \phi\left(N\left(x_{2 n}, y\right)\right)\right) \\
& \leq \psi\left(N\left(x_{2 n}, y\right)\right)
\end{aligned}
$$

Taking the upper limit in (3.2.31) and using (3.2.30) we get

$$
\psi\left(2 s^{3} b_{d}(z, G y)\right)=\psi\left(2 s^{4} \frac{1}{s} b_{d}(z, G y)\right) \leq \psi\left(s b_{d}(G y, z)\right)
$$

This implies $b_{d}(z, G y)=0$ and so $G y=z$. Thus $G y=T y=z$, so $y$ is a point 
of coincidence of the pair $(G, T)$.

Similarly we can show that $F v=S v=z$, so $v$ is a point of coincidence of the pair $(F, S)$. Therefore we have

$$
G y=T y=F v=S v=z .
$$

Let show that $z$ is a unique point of coincidence of pairs $(F, S)$ and $(G, T)$. Suppose that exists another point $z_{1} \in X$ such that $G y_{1}=T y_{1}=F v_{1}=S v_{1}=z_{1}$.

We consider,

$$
\begin{aligned}
N\left(z, z_{1}\right) & =\max \left\{b_{d}\left(S z, T z_{1}\right), b_{d}(F z, S z), b_{d}\left(G z_{1}, T z_{1}\right), b_{d}\left(S z, G z_{1}\right), b_{d}\left(F z, T z_{1}\right)\right\} \\
& =\max \left\{b_{d}\left(z, z_{1}\right), b_{d}(z, z), b_{d}\left(z_{1}, z_{1}\right), b_{d}\left(z, z_{1}\right), b_{d}\left(z, z_{1}\right)\right\} \\
& \leq 2 s b_{d}\left(z, z_{1}\right)
\end{aligned}
$$

Using contractive condition of theorem we have,

$$
\begin{aligned}
\psi\left(2 s^{4} b_{d}\left(z, z_{1}\right)\right) & =\psi\left(2 s^{4} b_{d}\left(F z, G z_{1}\right)\right) \\
& \leq f\left(\psi\left(N\left(z, z_{1}\right)\right), \phi\left(N\left(z, z_{1}\right)\right)\right) \\
& \leq \psi\left(N\left(z, z_{1}\right)\right) \\
& \leq \psi\left(2 s b_{d}\left(z, z_{1}\right)\right)
\end{aligned}
$$

The inequality above implies that $b_{d}\left(z, z_{1}\right)=0$ so $z=z_{1}$ that means the point of coincidence is unique.

Let prove that $z$ is a common fixed point. By the weak compatibility of the pairs $(F, S)$ and $(G, T)$ have: $S z=S F v=F S v=F z$ and $G z=G T y=T G y=T z$.

From condition of theorem we have,

$$
\begin{aligned}
\psi\left(2 s b_{d}(F z, G z)\right) & \leq \psi\left(2 s^{4} b_{d}(F z, G z)\right)=\psi\left(2 s^{4} b_{d}(F z, G z)\right) \\
& \leq f(\psi(N(z, z)), \phi(N(z, z))) \\
& \leq \psi(N(z, z))
\end{aligned}
$$

This inequality implies $2 s b_{d}(F z, G z) \leq N(z, z)$.

And

$$
\begin{aligned}
N(z, z) & =\max \left\{b_{d}(S z, T z), b_{d}(F z, S z), b_{d}(G z, T z), b_{d}(S z, G z), b_{d}(F z, T z)\right\} \\
& =\max \left\{b_{d}(F z, G z), b_{d}(F z, F z), b_{d}(G z, G z), b_{d}(F z, G z), b_{d}(F z, G z)\right\} \\
& \leq 2 s b_{d}(F z, G z)
\end{aligned}
$$

Again from (3.2.33) and (3.2.34) we get, $N(z, z)=2 s b_{d}(F z, G z)$.

By property of functions $\psi ; \phi$ and $C$-class, we have

$$
\begin{aligned}
& \psi\left(2 s b_{d}(F z, G z)\right) \leq f\left(\psi\left(2 s b_{d}(F z, G z)\right), \phi\left(2 s b_{d}(F z, G z)\right)\right) \\
& \leq \psi\left(2 s b_{d}(F z, G z)\right) \\
& \Rightarrow f\left(\psi\left(2 s b_{d}(F z, G z)\right), \phi\left(2 s b_{d}(F z, G z)\right)\right)=\psi\left(2 s b_{d}(F z, G z)\right) \\
& \Rightarrow \psi\left(2 s b_{d}(F z, G z)\right)=0 \text { or } \phi\left(2 s b_{d}(F z, G z)\right)=0 \\
& \Rightarrow 2 s b_{d}(F z, G z)=0 \Rightarrow b_{d}(F z, G z)=0
\end{aligned}
$$


So we obtained $b_{d}(F z, G z)=0$, that iz $F z=G z$. Therefore $F z=G z=S z=T z$. Let we prove that $z$ is a fixed point of $F$.

Again we consider

$$
\begin{aligned}
\psi\left(2 s b_{d}(F z, z)\right) & \leq \psi\left(2 s^{4} b_{d}(F z, z)\right)=\psi\left(2 s^{4} b_{d}(F z, G z)\right) \\
& \leq f(\psi(N(z, z)), \phi(N(z, z))) \\
& \leq \psi(N(z, z))
\end{aligned}
$$

By property of $\psi$ follows

$$
2 s b_{d}(F z, z) \leq N(z, z)
$$

where

$$
\begin{aligned}
N(z, z) & =\max \left\{b_{d}(S z, T z), b_{d}(F z, S z), b_{d}(G z, T z), b_{d}(S z, G z), b_{d}(F z, T z)\right\} \\
& =\max \left\{b_{d}(F z, z), b_{d}(F z, F z), b_{d}(z, z), b_{d}(F z, z), b_{d}(F z, z)\right\} \\
& \leq 2 s b_{d}(F z, z)
\end{aligned}
$$

From (3.2.36), (3.2.37) we get

$$
N(z, z)=2 s b_{d}(F z, z)
$$

In similar way as in (3.2.35) using (3.2.38), property of $C$-class and functions $\psi ; \phi$ we obtain, $b_{d}(F z, z)=0$ and $F z=z$. Hence $z$ is a common fixed point.

Uniqueness. Let we prove that the fixed point is unique. If suppose that $u$ and $z$ are two common fixed points of $F, G, S$, $T$ then from condition (b) we have,

$$
\begin{aligned}
\psi\left(2 s b_{d}(u, z)\right) & \leq \psi\left(2 s^{4} b_{d}(u, z)\right)=\psi\left(2 s^{4} b_{d}(F u, G z)\right) \\
& \leq f(\psi(N(u, z)), \phi(N(u, z))) \\
& \leq \psi(N(u, z))
\end{aligned}
$$

By property of $\psi$ we get $2 s b_{d}(u, z) \leq N(u, z)$. Also we have,

$$
\begin{aligned}
N(u, z) & =\max \left\{b_{d}(S u, T z), b_{d}(F u, S u), b_{d}(G z, T z), b_{d}(S u, G z), b_{d}(F u, T z)\right\} \\
& =\max \left\{b_{d}(u, z), b_{d}(u, u), b_{d}(z, z), b_{d}(u, z), b_{d}(u, z)\right\} \\
& \leq 2 s b_{d}(u, z)
\end{aligned}
$$

So, $N(u, z)=2 s b_{d}(u, z)$

and

$$
\begin{aligned}
& \psi\left(2 s b_{d}(u, z)\right) \leq f\left(\psi\left(2 s b_{d}(u, z)\right), \phi\left(2 s b_{d}(u, z)\right)\right) \leq \psi\left(2 s b_{d}(u, z)\right) \\
& \Rightarrow \psi\left(2 s b_{d}(u, z)\right)=0 \text { or } \phi\left(2 s b_{d}(u, z)\right)=0
\end{aligned}
$$

As a result $b_{d}(u, z)=0$ and so $u=z$.

The following is corollary of theorem 3.2 which is taken for parameter $s=1$ in a dislocated metric space.

Corollary 3.3 Let $\left(X, d_{l}\right)$ be a dislocated metric space and $S, T, F, G: X \rightarrow X$ are self-mappings such that (a) $F(X) \subset T(X)$, 
$F(X) \subset T(X)$ and exists $\psi \in \Psi, \phi \in \Phi$ and $f \in C$ such that satisfy the condition

$$
\psi\left(2 d_{l}(F x, G y)\right) \leq f(\psi(N(x, y)), \phi(N(x, y)))
$$

for all $x, y \in X$, where $N(x, y)$ is defined as in (3.1.0). If one of $F(X), S(X), T(X)$ or $G(X)$ is a complete subspace of $X$, then $(F, S)$ and $(G, T)$ have a point of coincidence in $X$. Moreover if suppose that $(F, S)$ and $(G, T)$ are weakly compatible pairs, then $F, G, S, T$ have a unique common fixed point.

Now we give an example to support our Theorem 3.2.

Example 3.4 Let $X=[0, \infty)$ and $b_{d}(x, y)=(x+y)^{2}$. Then the pair $\left(X, b_{d}\right)$ is a $b$-dislocated metric space with parameter $s=2$. We define the functions $F, G, S$ and $T$ as follows: $S x=\frac{2 x}{5}, T x=\frac{x}{2}, F x=\frac{x}{30}, G x=\frac{x}{24}$. The pairs $(S, F)$ and $(T, G)$ are weakly compatible, functions $F, G, S$ and $T$ are continuous and $F(X) \subset T(X), G(X) \subset S(X)$

We have,

$$
\begin{aligned}
& \psi\left(2 s^{4} b_{d}(F x, G y)\right) \\
& =\psi\left(32 b_{d}\left(\frac{x}{30}, \frac{y}{24}\right)\right)=\psi\left(32\left(\frac{x}{30}+\frac{y}{24}\right)^{2}\right)=\psi\left(\frac{32}{36}\left(\frac{x}{5}+\frac{y}{4}\right)^{2}\right) \\
& =\psi\left(\frac{8}{9} \frac{(4 x+5 y)^{2}}{400}\right)=\psi\left(\frac{2}{9} \frac{(4 x+5 y)^{2}}{100}\right)=\psi\left(\frac{2}{9}\left(\frac{2 x}{5}+\frac{y}{2}\right)^{2}\right) \\
& \leq \psi\left(\frac{1}{4}\left(\frac{2 x}{5}+\frac{y}{2}\right)^{2}\right)=\psi\left(\frac{1}{4} b_{d}(S x, T y)\right)=\frac{1}{4} b_{d}(S x, T y) \leq \frac{1}{4} M(x, y) \\
& =M(x, y)-\frac{3}{4} M(x, y)=\psi(M(x, y))-\phi(M(x, y)) \\
& =f(\psi(M(x, y)), \phi(M(x, y)))
\end{aligned}
$$

where $f(s, t)=s-t ; \psi(t)=t$ and $\phi(t)=\frac{3}{4} t$, for all $x, y \in X$.

Thus all conditions of theorem 3.2 are satisfied and $x=0$ is the unique common fixed point of $S, T, F$ and $G$.

In a similar way as in Theorem 3.2, the following theorem can be proved.

Theorem 3.5 Let $\left(X, b_{d}\right)$ be a complete $b$-dislocated metric space with parameter $s \geq 1$ and $S, T, F, G: X \rightarrow X$ are self-mappings such that (a) $F(X) \subset T(X), G(X) \subset S(X)$ and satisfy generalized $f-(\psi, \phi, s)$ weakly contractive condition. If one of $F(X), S(X), T(X)$ or $G(X)$ is closed, then $(F, S)$ and $(G, T)$ have a point of coincidence in $X$. Moreover if suppose that $(F, S)$ and $(G, T)$ are weakly compatible pairs, then $F, G, S, T$ have a unique common fixed point.

For the different functions $f$ of $C$-class (refer to example 2.15) we can take the following corollaries. 
Corollary 3.6 Let $\left(X, b_{d}\right)$ be a $b_{d}$-dislocated metric space with parameter $s \geq 1$ and $S, T, F, G: X \rightarrow X$ are self-mappings where (a) $F(X) \subset T(X), G(X) \subset S(X)$ and exists $\psi \in \Psi, \phi \in \Phi$ such that satisfies the condition

$$
\psi\left(2 s^{4} b_{d}(F x, G y)\right) \leq \psi(N(x, y))-\phi(N(x, y))
$$

for all $x, y \in X$, where $N(x, y)$ is defined as in (3.1.0). If one of $F(X), S(X), T(X)$ or $G(X)$ is a complete subspace of $X$, then $(F, S)$ and $(G, T)$ have a point of coincidence in $X$. Moreover if suppose that $(F, S)$ and $(G, T)$ are weakly compatible pairs, then $F, G, S, T$ have a unique common fixed point.

Proof. If we take in Theorem 3.2 the function $f$ as $f(s, t)=s-t$ then we get the corollary.

Corollary 3.7 Let $\left(X, b_{d}\right)$ be a $b_{d}$-dislocated metric space with parameter $s \geq 1$ and $S, T, F, G: X \rightarrow X$ are self-mappings where (a)

$F(X) \subset T(X), G(X) \subset S(X)$ and exists $\psi \in \Psi, \phi \in \Phi$ such that satisfies the condition

$$
\psi\left(2 s^{4} b_{d}(F x, G y)\right) \leq \frac{\psi(N(x, y))-\phi(N(x, y))}{1+\phi(N(x, y))}
$$

for all $x, y \in X$, where $N(x, y)$ is defined as in (3.1.0). If one of $F(X), S(X), T(X)$ or $G(X)$ is a complete subspace of $X$, then $(F, S)$ and $(G, T)$ have a point of coincidence in $X$. Moreover if suppose that $(F, S)$ and $(G, T)$ are weakly compatible pairs, then $F, G, S, T$ have a unique common fixed point.

Proof. This corollary is obtained from Theorem 3.2 if we take as $f$ the function $f(s, t)=\frac{s-t}{1+t}$.

Corollary 3.8 Let $\left(X, b_{d}\right)$ be a $b_{d}$-dislocated metric space with parameter $s \geq 1$ and $S, T, F, G: X \rightarrow X$ are self-mappings where (a)

$F(X) \subset T(X), G(X) \subset S(X)$ and exists $\psi \in \Psi, \phi \in \Phi$ such that satisfies the condition

$$
\psi\left(2 s^{4} b_{d}(F x, G y)\right) \leq \frac{\psi(N(x, y))}{1+\phi(N(x, y))}
$$

for all $x, y \in X$, where $N(x, y)$ is defined as in (3.1.0). If one of $F(X), S(X), T(X)$ or $G(X)$ is a complete subspace of $X$, then $(F, S)$ and $(G, T)$ have a point of coincidence in $X$. Moreover if suppose that $(F, S)$ and $(G, T)$ are weakly compatible pairs, then $F, G, S, T$ have a unique common fixed point.

Proof. If we take in Theorem 3.2 the function $f$ as $f(s, t)=\frac{s}{1+t}$ then we get the corollary.

Corollary 3.9 Let $\left(X, b_{d}\right)$ be a $b_{d}$-dislocated metric space with parameter 
$s \geq 1$ and $S, T, F, G: X \rightarrow X$ are self-mappings where (a)

$F(X) \subset T(X), G(X) \subset S(X)$ and exists $\psi \in \Psi, \phi \in \Phi$ such that satisfies the condition

$$
\psi\left(2 s^{4} b_{d}(F x, G y)\right) \leq \frac{\psi(N(x, y)) \phi(N(x, y))}{1+\phi(N(x, y))}
$$

for all $x, y \in X$, where $N(x, y)$ is defined as in (3.1.0). If one of $F(X), S(X), T(X)$ or $G(X)$ is a complete subspace of $X$, then $(F, S)$ and $(G, T)$ have a point of coincidence in $X$. Moreover if suppose that $(F, S)$ and $(G, T)$ are weakly compatible pairs, then $F, G, S, T$ have a unique common fixed point.

Proof. If we take in Theorem 3.2 the function $f$ as $f(s, t)=\frac{s t}{1+t}$ then we get the corollary.

Remark 3.10 As a consequence of theorem 3.2 and all corollaries for taking

1) the parameter $s=1$.

2) the parameter $s=1$ and $G=F$ and $T=S$.

3) functions $f$ from the set $C$ and taking $\psi(t)=t$ and $\phi(t)=(1-k) t$.

We can establish many other corollaries in the setting of dislocated and $b$-dislocated metric spaces.

4) Our results unify, generalize, and extend several ones obtained earlier in a lot of papers concerning $b$-metric, dislocated and $b$-dislocated metric spaces (as in references [13] [15] [25] [26] [30] [31]).

In this section, we use the notion of $T$-contractions introduced by Beiranvad et al. in [3] as a new class of contractive mappings, by generalizing the contractive condition in terms of another function. These contractions have been used by many authors. In this direction in order to generalize some other well-known results as in [32] [33] [34] we extend the notion of $f-(\psi, \phi, s)$ generalized weak contractions in the context of $T$-contractions, giving the following theorem.

Theorem 3.11 Let $\left(X, b_{d}\right)$ be a complete $b$-dislocated metric space with parameter $s \geq 1$ and $T: X \rightarrow X$ be an injective, continuous and sequentially convergent mapping. Let $F, G: X \rightarrow X$ be self-mappings and if exist $\psi \in \Psi$, $\phi \in \Phi$ and $f \in C$ such that

$$
\psi\left(2 s^{4} b_{d}(T F x, T G y)\right) \leq f(\psi(N(T x, T y)), \phi(N(T x, T y)))
$$

for all $x, y \in X$, where

$$
\begin{aligned}
& N(T x, T y) \\
& =\max \left\{b_{d}(T x, T y), b_{d}(T x, T F x), b_{d}(T y, T G y), \frac{b_{d}(T x, T G y)+b_{d}(T y, T F x)}{4 s}\right\}
\end{aligned}
$$

then $F, G$ have a unique common fixed point.

Proof. We divide the proof into two parts as follows.

First part. Each fixed point $u$ of $F$ is a fixed point of $G$ and conversely, and the 
common fixed point of $F, G$ is unique.

Let $u \in X$ be a fixed point of $F$. If $b_{d}(F u, G u)=0$ then, follows that $u=F u=G u$ and so $u$ is a fixed point of $G$. If we suppose that $b_{d}(F u, G u)>0$, we evaluate $N(T u, T u)$ as;

$$
\begin{aligned}
& N(T u, T u) \\
& =\max \left\{b_{d}(T u, T u), b_{d}(T u, T F u), b_{d}(T u, T G u), \frac{b_{d}(T u, T G u)+b_{d}(T u, T F u)}{2 s}\right\} \\
& =\max \left\{b_{d}(T u, T u), b_{d}(T u, T u), b_{d}(T u, T G u), \frac{b_{d}(T u, T G u)+b_{d}(T u, T u)}{4 s}\right\} \\
& \leq 2 s b_{d}(T u, T G u)
\end{aligned}
$$

So we have

$$
N(T u, T u) \leq 2 s b_{d}(T u, T G u)
$$

Then by contractive condition (B), we have

$$
\begin{aligned}
\psi\left(2 s b_{d}(T u, T G u)\right) & \leq \psi\left(2 s^{2} b_{d}(T F u, T G u)\right) \\
& \leq f(\psi(N(T u, T u)), \phi(N(T u, T u))) \\
& \leq \psi(N(T u, T u))
\end{aligned}
$$

By property of $\psi$ we have

$$
2 s b_{d}(T u, T G u) \leq N(T u, T u)
$$

Hence from (3.11.1) and (3.11.2) follows $N(T u, T u)=2 s b_{d}(T u, T G u)$. Again

$$
\begin{aligned}
& \psi\left(2 s b_{d}(T u, T G u)\right) \leq \psi\left(2 s^{2} b_{d}(T F u, T G u)\right) \\
& \leq f(\psi(N(T u, T u)), \phi(N(T u, T u))) \\
& =f\left(\psi\left(2 s b_{d}(T u, T G u)\right), \phi\left(2 s b_{d}(T u, T G u)\right)\right) \\
& \leq \psi\left(2 s b_{d}(T u, T G u)\right) \\
& \Rightarrow f\left(\psi\left(2 s b_{d}(T u, T G u)\right), \phi\left(2 s b_{d}(T u, T G u)\right)\right)=\psi\left(2 s b_{d}(T u, T G u)\right) \\
& \Rightarrow \psi\left(2 s b_{d}(T u, T G u)\right)=0 \text { or } \phi\left(2 s b_{d}(T u, T G u)\right)=0
\end{aligned}
$$

By property of $\psi, \phi$ we get $b_{d}(T u, T G u)=0$ that is $T u=T G u$ and by injectivity of $T$ follows $u=G u$.

Thus $u$ is a fixed point of $G$. Similarly we can prove the other implication.

Second part. We prove that the function $F$ has a fixed point. We define two eterative sequences $\left\{x_{n}\right\}$ as $x_{2 n+1}=f x_{2 n}$ and $x_{2 n+2}=g x_{2 n+1}$, and $\left\{y_{n}\right\}$ as $y_{n}=T x_{n}$ for each $n=0,1,2, \cdots$.

If for some $n$, we have $x_{2 n+1}=x_{2 n}$ then $x_{2 n}=x_{2 n+1}=f x_{2 n}$ and $x_{2 n}$ is a fixed point of $F$ and by the first part $x_{2 n}$ is a fixed point of $G$ and the proof is completed.

Now, we assume that $x_{2 n+1} \neq x_{2 n}$ for all $n$, and since $T$ is injective we have $T x_{2 n+1} \neq T x_{2 n}$; then from condition (B) of theorem, we have 


$$
\begin{aligned}
\psi\left(2 s b_{d}\left(T x_{2 n+1}, T x_{2 n}\right)\right) & \leq \psi\left(2 s^{2} b_{d}\left(T x_{2 n+1}, T x_{2 n}\right)\right)=\psi\left(2 s b_{d}\left(T f x_{2 n}, T g x_{2 n-1}\right)\right) \\
& \leq f\left(\psi\left(N\left(T x_{2 n}, T x_{2 n-1}\right)\right), \phi\left(N\left(T x_{2 n}, T x_{2 n-1}\right)\right)\right) \\
& \leq \psi\left(N\left(T x_{2 n}, T x_{2 n-1}\right)\right)
\end{aligned}
$$

where

$$
\begin{aligned}
& N\left(T x_{2 n}, T x_{2 n-1}\right) \\
&= \max \left\{b_{d}\left(T x_{2 n}, T x_{2 n-1}\right), b_{d}\left(T x_{2 n}, T f x_{2 n}\right), b_{d}\left(T x_{2 n-1}, T g x_{2 n-1}\right),\right. \\
&\left.\frac{b_{d}\left(T x_{2 n}, T g x_{2 n-1}\right)+b_{d}\left(T x_{2 n-1}, T f x_{2 n}\right)}{4 s}\right\} \\
&= \max \left\{b_{d}\left(T x_{2 n}, T x_{2 n-1}\right), b_{d}\left(T x_{2 n}, T x_{2 n+1}\right), b_{d}\left(T x_{2 n-1}, T x_{2 n}\right),\right. \\
& \leq \max \left\{b_{d}\left(T x_{2 n}, T x_{2 n-1}\right), b_{d}\left(T x_{2 n}, T x_{2 n+1}\right), b_{d}\left(T x_{2 n-1}, T x_{2 n}\right),\right. \\
&\left.\frac{b_{d}\left(T x_{2 n}, T x_{2 n}\right)+b_{d}\left(T x_{2 n-1}, T x_{2 n+1}\right)}{4 s}\right\} \\
&
\end{aligned}
$$

If $b_{d}\left(T x_{2 n}, T x_{2 n-1}\right)<b_{d}\left(T x_{2 n+1}, T x_{2 n}\right)$ then from (3.11.3) we get

$$
N\left(T x_{2 n}, T x_{2 n-1}\right) \leq b_{d}\left(T x_{2 n+1}, T x_{2 n}\right)
$$

Using condition (B) and property of C-class, we have

$$
\begin{aligned}
\psi\left(b_{d}\left(T x_{2 n+1}, T x_{2 n}\right)\right) & \leq \psi\left(2 s^{2} b_{d}\left(T x_{2 n+1}, T x_{2 n}\right)\right) \\
& =\psi\left(2 s^{2} b_{d}\left(T x_{2 n}, T g x_{2 n-1}\right)\right) \\
& \leq f\left(\psi\left(N\left(T x_{2 n}, T x_{2 n-1}\right)\right), \phi\left(N\left(T x_{2 n}, T x_{2 n-1}\right)\right)\right) \\
& \leq \psi\left(N\left(T x_{2 n}, T x_{2 n-1}\right)\right)
\end{aligned}
$$

By property of function $\psi$ we have

$$
b_{d}\left(T x_{2 n+1}, T x_{2 n}\right) \leq N\left(T x_{2 n}, T x_{2 n-1}\right)
$$

From (3.11.4) and (3.11.5) we get

$$
N\left(T x_{2 n}, T x_{2 n-1}\right)=b_{d}\left(T x_{2 n+1}, T x_{2 n}\right)
$$

Also from condition of theorem and (3.11.6), we have

$$
\begin{aligned}
\psi\left(b_{d}\left(T x_{2 n+1}, T x_{2 n}\right)\right) & \leq \psi\left(2 s^{2} b_{d}\left(T x_{2 n+1}, T x_{2 n}\right)\right) \\
& =\psi\left(2 s^{2} b_{d}\left(T x_{2 n}, T g x_{2 n-1}\right)\right) \\
& \leq f\left(\psi\left(N\left(T x_{2 n}, T x_{2 n-1}\right)\right), \phi\left(N\left(T x_{2 n}, T x_{2 n-1}\right)\right)\right) \\
& =f\left(\psi\left(b_{d}\left(T x_{2 n+1}, T x_{2 n}\right)\right), \phi\left(b_{d}\left(T x_{2 n+1}, T x_{2 n}\right)\right)\right) \\
& \leq \psi\left(b_{d}\left(T x_{2 n+1}, T x_{2 n}\right)\right)
\end{aligned}
$$

Also we have, 


$$
\begin{aligned}
& \begin{aligned}
\psi\left(b_{d}\left(T x_{2 n+1}, T x_{2 n}\right)\right) & \leq f\left(\psi\left(b_{d}\left(T x_{2 n+1}, T x_{2 n}\right)\right), \phi\left(b_{d}\left(T x_{2 n+1}, T x_{2 n}\right)\right)\right) \\
& \leq \psi\left(b_{d}\left(T x_{2 n+1}, T x_{2 n}\right)\right)
\end{aligned} \\
& \Rightarrow f\left(\psi\left(b_{d}\left(T x_{2 n+1}, T x_{2 n}\right)\right), \phi\left(b_{d}\left(T x_{2 n+1}, T x_{2 n}\right)\right)\right)=\psi\left(b_{d}\left(T x_{2 n+1}, T x_{2 n}\right)\right) \\
& \Rightarrow \psi\left(b_{d}\left(T x_{2 n+1}, T x_{2 n}\right)\right)=0 \text { or } \phi\left(b_{d}\left(T x_{2 n+1}, T x_{2 n}\right)\right)=0
\end{aligned}
$$

By property of $\psi$ and $\phi$ we have $b_{d}\left(T x_{2 n+1}, T x_{2 n}\right)=0$ so $T x_{2 n+1}=T x_{2 n}$ which is a contradiction since we supposed $T x_{2 n+1} \neq T x_{2 n}$.

Hence, we have

$$
b_{d}\left(T x_{2 n+1}, T x_{2 n}\right) \leq b_{d}\left(T x_{2 n}, T x_{2 n-1}\right) .
$$

Similarly, we have that

$$
b_{d}\left(T x_{2 n+2}, T x_{2 n+1}\right) \leq b_{d}\left(T x_{2 n+1}, T x_{2 n}\right)
$$

Therefore for all $n$ we have

$$
b_{d}\left(T x_{n+1}, T x_{n}\right) \leq b_{d}\left(T x_{n}, T x_{n-1}\right)
$$

and $\left\{b_{d}\left(T x_{n+1}, T x_{n}\right)\right\}$ is a non increasing sequence of nonnegative real numbers and bounded below. Hence there exists $r \geq 0$ such that

$$
\lim _{n \rightarrow \infty} b_{d}\left(y_{n+1}, y_{n}\right)=\lim _{n \rightarrow \infty} N\left(T x_{n+1}, T x_{n}\right)=r
$$

By the property of functions $\psi$ and $\phi$, we have

$$
\psi(r)=\lim _{n \rightarrow \infty} \psi\left(N\left(T x_{n+1}, T x_{n}\right)\right) \text { and } \phi(r) \leq \liminf _{n \rightarrow \infty} \phi\left(N\left(T x_{n+1}, T x_{n}\right)\right)
$$

If we consider condition (B) we have,

$$
\begin{aligned}
\psi\left(b_{d}\left(T x_{n+1}, T x_{n}\right)\right) & \leq \psi\left(2 s^{2} b_{d}\left(T x_{n+1}, T x_{n}\right)\right) \\
& \leq f\left(\psi\left(N\left(T x_{n}, T x_{n-1}\right)\right), \phi\left(N\left(T x_{n}, T x_{n-1}\right)\right)\right) \\
& \leq \psi\left(N\left(T x_{n}, T x_{n-1}\right)\right)
\end{aligned}
$$

Taking the upper limit as $n \rightarrow \infty$ in (3.11.8) and using (3.11.7) we have that,

$$
\begin{aligned}
& \psi(r) \leq f(\psi(r), \phi(r)) \leq \psi(r) \Rightarrow f(\psi(r), \phi(r))=\psi(r) \\
& \Rightarrow \psi(r)=0 \text { or } \phi(r)=0 .
\end{aligned}
$$

From (3.11.9) and property of $\psi$ and $\phi$ follows that $r=0$ and also

$$
\lim _{n \rightarrow \infty} b_{d}\left(y_{n+1}, y_{n}\right)=\lim _{n \rightarrow \infty} N\left(T x_{n+1}, T x_{n}\right)=0
$$

In a similar way as in Theorem 3.2 we can show that the sequence $\left\{T x_{n}\right\}$ (also $\left.\left\{y_{n}\right\}\right)$ is a $b_{d}$-Cauchy sequence in b-dislocated metric space $\left(X, b_{d}\right)$. Since $X$ is complete there exists $z \in X$ such that $\lim _{n \rightarrow \infty} y_{n}=\lim _{n \rightarrow \infty} T x_{n}=z$. Since $T$ is sequentially convergent, we can deduce that $\left\{x_{n}\right\}^{n \rightarrow \infty}$ is convergent to $u \in X$ and the subsequences $\left\{F x_{2 n}\right\},\left\{G x_{2 n+1}\right\}$ converge to $u$, that means

$$
\lim _{n \rightarrow \infty} b_{d}\left(y_{2 n}, u\right)=0 \text { and } \lim _{n \rightarrow \infty} b_{d}\left(y_{2 n+1}, u\right)=0 .
$$

Since $T$ is continuous we have $T u=\lim _{n \rightarrow \infty} T x_{n}=\lim _{n \rightarrow \infty} y_{n}=z$.

Let we prove that $u$ is a fixed point of $F$ and $\stackrel{n \rightarrow \infty}{G}(F u=G u=u)$. If suppose that $u \neq F u$ then since $T$ is injective follows $z=T u \neq T F u$ (and $b_{d}(T u, T F u)>0$ ) 
Consider,

$$
\begin{gathered}
N\left(T u, T x_{2 n-1}\right)=\max \left\{b_{d}\left(T u, T x_{2 n-1}\right), b_{d}(T u, T f u), b_{d}\left(T x_{2 n-1}, T g x_{2 n-1}\right),\right. \\
\left.\frac{b_{d}\left(T u, T g x_{2 n-1}\right)+b_{d}\left(T x_{2 n-1}, T f u\right)}{4 s}\right\} \\
=\max \left\{b_{d}\left(T u, y_{2 n-1}\right), b_{d}(T u, T f u), b_{d}\left(y_{2 n-1}, y_{2 n}\right),\right. \\
\left.\frac{b_{d}\left(T u, y_{2 n}\right)+b_{d}\left(y_{2 n-1}, T f u\right)}{4 s}\right\}
\end{gathered}
$$

Taking the upper limit in (3.11.11) and using lemma (2.13), and result (3.11.10) we get

$$
\lim _{n \rightarrow \infty} \sup N\left(T u, T x_{2 n-1}\right) \leq \max \left\{0, b_{d}(T u, T f u), 0, \frac{s b_{d}(T u, T f u)}{4 s}\right\}=b_{d}(T u, T f u)
$$

According to contractive condition (B) we have,

$$
\begin{aligned}
\psi\left(2 s^{2} b_{d}\left(T F u, T x_{2 n}\right)\right) & =\psi\left(2 s^{2} b_{d}\left(T F u, T G x_{2 n-1}\right)\right) \\
& \leq f\left(\psi\left(N\left(T u, T x_{2 n-1}\right)\right), \phi\left(N\left(T u, T x_{2 n-1}\right)\right)\right) \\
& \leq \psi\left(N\left(T u, T x_{2 n-1}\right)\right)
\end{aligned}
$$

Taking the upper limit in (3.11.13) and using lemma (2.13), we obtain,

$$
\psi\left(2 s b_{d}(T F u, T u)\right)=\psi\left(2 s^{2} \frac{1}{s} b_{d}(T F u, T u)\right) \leq \psi\left(s b_{d}(T F u, T u)\right)
$$

This implies that $b_{d}(T u, T F u)=0$ that is $T u=T F u$ which is a contradiction. As a result $u=F u$ and $u$ is a fixed point of $F$. By the first part of proof $u$ is a fixed point of $G$ and also a common fixed point.

Easily using the contractive condition (B) of theorem can be proved that the common fixed point is unique.

Example 3.12 Let $X=[0, \infty)$ be equipped with the $b$-dislocated metric $b_{d}(x, y)=(x+y)^{2}$ for all $x, y \in X$, where $s=2$. It is clear that $\left(X, b_{d}\right)$ is a complete $b$-dislocated metric space. Also let be the self-mappings $T, F, G: X \rightarrow X$ defined by $T(x)=\frac{x}{2}, F(x)=\frac{x}{5}, G(x)=\frac{x}{10}$. We note, $T$ is continuous and sequentially convergent.

$$
\begin{aligned}
& \text { If } \psi(t)=t, \phi(t)=\frac{17}{25} t \text { and } f(s, t)=s-t \text { then for each } x, y \in X \text {, we have } \\
& \psi\left(2 s^{2} b_{d}(T F x, T G y)\right)=2 s^{2} b_{d}(T F x, T G y)=8 b_{d}\left(\frac{x}{10}, \frac{y}{20}\right)=8\left(\frac{x}{10}+\frac{y}{20}\right)^{2} \leq 8\left(\frac{x}{10}+\frac{y}{10}\right)^{2} \\
& =\frac{8}{25} \frac{(x+y)^{2}}{4}=\frac{8}{25}\left(\frac{x}{2}+\frac{y}{2}\right)^{2}=\frac{8}{25} b_{d}(T x, T y) \leq \frac{8}{25} N(T x, T y) \\
& =N(T x, T y)-\frac{17}{25} N(T x, T y)=\psi(N(T x, T y))-\phi(N(T x, T y)) \\
& =f(\psi(N(T x, T y)), \phi(N(T x, T y)))
\end{aligned}
$$


Thus $T, F, G$ satisfy all the conditions of Theorem 3.11. Moreover $x=0$ is the unique common fixed point of $F, G$.

If in theorem3.11 we take $F=G$ we get the following corollary.

Corollary 3.13 Let $\left(X, b_{d}\right)$ be a complete $b$-dislocated metric space with parameter $s \geq 1$ and $T, G: X \rightarrow X$ be two self mappings, where $T$ is injective, continuous and sequentially convergent. If exist $\psi \in \Psi, \phi \in \Phi$ and $f \in C$ such that

$$
\psi\left(2 s^{2} b_{d}(T G x, T G y)\right) \leq f(\psi(N(T x, T y)), \phi(N(T x, T y)))
$$

for all $x, y \in X$, where

$$
\begin{aligned}
& N(T x, T y) \\
& =\max \left\{b_{d}(T x, T y), b_{d}(T x, T G x), b_{d}(T y, T G y), \frac{b_{d}(T x, T G y)+b_{d}(T y, T G x)}{4 s}\right\}
\end{aligned}
$$

then $G$ has a unique fixed point.

Corollary 3.14 Let $\left(X, b_{d}\right)$ be a complete $b$-dislocated metric space with parameter $s \geq 1$ and $T: X \rightarrow X$ be an injective, continuous and sequentially convergent mapping. Let $F, G: X \rightarrow X$ be self-mappings and if exist $\psi \in \Psi$, $\phi \in \Phi$ such that

$$
\psi\left(2 s^{4} b_{d}(T F x, T G y)\right) \leq \psi(N(T x, T y))-\phi(N(T x, T y))
$$

for all $x, y \in X$, where

$$
\begin{aligned}
& N(T x, T y) \\
& =\max \left\{b_{d}(T x, T y), b_{d}(T x, T F x), b_{d}(T y, T G y), \frac{b_{d}(T x, T G y)+b_{d}(T y, T F x)}{4 s}\right\}
\end{aligned}
$$

then $F, G$ have a unique common fixed point.

Proof. If we take in Theorem 3.11 the function $f \in C$ as $f(s, t)=s-t$ then we get the corollary.

\section{Remark 3.15}

1) Theorem 3.11 generalizes, extends and unifies results as Theorem 8 in [32], Theorem 4 in [33] and many existing results of literature in a set effective larger as $b$-dislocated metric spaces.

2) The class $C$ of functions has a general character and so according to example 2.15, we can provide many results from theorem 3.11.

3) If we take in theorem 3.11 the parameter $s=1$ as a consequence, we obtain results in a dislocated metric space.

\section{References}

[1] Hitzler, P. and Seda, A.K. (2000) Dislocated Topologies. Journal of Electrical Engineering, 51, 3-7.

[2] Aage, C.T. and Salunke, J.N. (2008) Some Results of Fixed Point Theorem in Dislocated Quasi-Metric Spaces. Bulletin of Marathwada Mathematical Society, 9, 1-5.

[3] Beiranvand, A., Moradi, S., Omid, M. and Pazandeh, H. (2009) Two Fixed-Point 
Theorems for Special Mapping. https://arxiv.org/abs/0903.1504

[4] Jungck, G. (1986) Compatible Mappings and Common Fixed Points. International Journal of Mathematics and Mathematical Sciences, 9, 771-779. https://doi.org/10.1155/S0161171286000935

[5] Shrivastava, R., Ansari, Z.K. and Sharma, M. (2012) Some Results on Fixed Points in Dislocated and Dislocated Quasi-Metric Spaces. Journal of Advanced Studies in Topology, 3, 25-31.

[6] Kutbi, M.A., Arshad, M., Ahmad, J. and Azam, A. (2014) Generalized Common Fixed Point Results with Applications. Abstract and Applied Analysis, 2014, Article ID: 363925.

[7] Aydi, H., Vetro, C., Sintunavarat, W. and Kumam, P. (2012) Coincidence and Fixed Points for Contractions and Cyclical Contractions in Partial Metric Spaces. Fixed Point Theory and Applications, 2012, 124.

[8] Czerwik, S. (1993) Contraction Mappings in b-Metric Spaces. Acta Mathematica et Informatica Universitatis Ostraviensis, 1, 5-11.

[9] Ciric'c, L.J. (1972) Fixed Points for Generalized Multi-Valued Contractions. Matematički Vesnik, 9, 265-272.

[10] Ciric, L.B. (1974) A Generalization of Banach's Contraction Principle. Proceedings of the American Mathematical Society, 45, 267-273.

https://doi.org/10.2307/2040075

[11] Das, K.M. and Naik, K.V. (1979) Common Fixed Point Theorems for Commuting Maps on Metric Spaces. Proceedings of the American Mathematical Society, 77, 369-373.

[12] Arshad, M., Shoaib, A. and Beg, I. (2013) Fixed Point of a Pair of Contractive Dominated Mappings on a Closed Ball in an Ordered Dislocated Metric Space. Fixed Point Theory and Applications, 2013, 115. https://doi.org/10.1186/1687-1812-2013-115

[13] Zoto, K. (2013) Weakly Compatible Mappings and Fixed Points in Dislocated Metric Spaces. International Journal of Mathematical Archive, 4, 131-137.

[14] Beg, I., Arshad, M. and Shoaib, A. (2015) Fixed Point on a Closed Ball in Ordered Dislocated Quasi Metric Spaces. Fixed Point Theory, 16, 195-206.

[15] Hussain, N., Roshan, J.R., Parvaneh, V. and Abbas, M. (2013) Common Fixed Point Results for Weak Contractive Mappings in Ordered $b$-Dislocated Metric Spaces with Applications. Journal of Inequalities and Applications, 2013, 486.

[16] Bakhtin, I.A. (1989) The Contraction Mapping Principle in Quasi-Metric Spaces. Functional Analysis, 30, 26-37.

[17] Khan, M.S., Swaleh, M. and Sessa, S. (1984) Fixed Points Theorems by Altering Distances between the Points. Bulletin of the Australian Mathematical Society, 30, 1-9. https://doi.org/10.1017/S0004972700001659

[18] Popa, V. and Mocanu, M. (2009) Altering Distances and Common Fixed Points under Implicit Relations. Hacettepe Journal of Mathematics and Statistics, 38, 329-337.

[19] Sastri, K.P. and Babu, G.V.R. (1998) Fixed Point Theorems in Metric Spaces by Altering Distance. Bulletin of the Calcutta Mathematical Society, 90, 175-182.

[20] Sastri, K.P. and Babu, G.V.R. (1999) Some Fixed Point Theorems by Altering Distances between Two Points. Indian Journal of Pure and Applied Mathematics, 30, 641-647. 
[21] Babu, G.V.R., Nageswara Rao, K. and Alemayehu, G.N. (2010) Common Fixed Points of Two Pair of Generalized Weakly Contractive Maps. Advanced Studies in Contemporary Mathematics, 20, 575-594.

[22] Babu, G.V.R. and Alemayehu, G.N. (2010) Point of Coincidence and Common Fixed Points of a Pair of Generalized Weakly Contractive Maps. Journal of Advanced Research in Pure Mathematics, 2, 89-106. https://doi.org/10.5373/jarpm.338.010810

[23] Choudhury, B.S., Konar, P., Rhoades, B.E. and Metiya, N. (2011) Fixed Point Theorems for Generalized Weak Contractive Mappings. Nonlinerar Analysis, 74, 2116-2126. https://doi.org/10.1016/j.na.2010.11.017

[24] Ansari, A.H. (2014) Note on $(\psi, \varphi)$-Contractive Type Mappings and Related Fixed Point. The 2nd Regional Conference on Mathematics and Applications, Tehran, September 2014, 377-380.

[25] Hoxha, E., Ansari, A.H. and Zoto, K. (2014) Some Common Fixed Point Results through Generalized Altering Distances on Dislocated Metric Spaces. Proceedings of the 3rd Electronic International Interdisciplinary Conference, Zilina, 1-5 September 2014, 403-409.

[26] Karapinar, E. and Salimi, P. (2013) Dislocated Metric Space to Metric Spaces with Some Fixed Point Theorems. Fixed Point Theory and Applications, 2013, 222. https://doi.org/10.1186/1687-1812-2013-222

[27] Doric, D. (2009) Common Fixed Point for Generalized ( $\psi, \varphi)$-Weak Contractions. Applied Mathematics Letters, 22, 1896-1900. https://doi.org/10.1016/j.aml.2009.08.001

[28] Dutta, P.N. and Choudhury, B.S. (2008) A Generalisation of Contraction Principle in Metric Spaces. Fixed Point Theory and Applications, 2008, Article ID: 406368. https://doi.org/10.1155/2008/406368

[29] Kumari, P.S. (2012) Common Fixed Point Theorems on Weakly Compatible Maps on Dislocated Metric Spaces. Mathematical Sciences, 6, 71. https://doi.org/10.1186/2251-7456-6-71

[30] He, F. (2015) Common Fixed Point of Four Self Maps on Dislocated Metric Spaces. Journal of Nonlinear Sciences and Applications, 8, 301-308. https://doi.org/10.22436/jnsa.008.04.02

[31] Bennani, S., Bourijal, H., El Moutawakil, D. and Mhanna, S. (2015) Some Common Fixed Point Theorems in Dislocated Metric Spaces. Journal of Nonlinear Sciences and Applications, 8, 86-92. https://doi.org/10.22436/jnsa.008.02.01

[32] An, T.V., Chi, K.P., Karapınarand, E. and Thanh, T.D. (2012) An Extension of Generalized $\psi, \phi$-Weak Contractions. International Journal of Mathematics and Mathematical Sciences, 2012, Article ID: 431872. https://doi.org/10.1155/2012/431872

[33] Mustafa, Z., Roshan, J.R., Parvaneh, V. and Kadelburg, Z. (2013) Some Common Fixed Point Results in Ordered b-Metric Spaces. Journal of inequalities and Applications, 2013, 562.

[34] Moradi, S. and Omid, M. (2010) A Fixed-Point Theorem for Integral Type Inequality Depending on Another Function. International Journal of Mathematical Analysis, 4, 1491-1499. 